\title{
PARASITOSIS INTESTINAL EN TRES GRUPOS DE LA POBLACION DE MANTA
}

\author{
Dr. l'E' IX PFRE'? C.
}

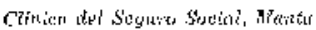

La parasitnois inteptinal constituye, on nuostro redio, una do las caumar primordiales jor las euales lis pacierLes acutcon in lat tousulta méticà adomás. éerto 1jpo do parasitosis so ha const:tuída en un mal endémieo, de. biclo en géan parte a dos ractorest-i: 10-Las molas condiciunex highéricias y sanitarias del medic ambiente en dy:te vive la stayos parte de nuestro pucblo: escascz de agua potilble, folto de cárнizacion, malas condiciones de la vi" vienda, abundancia re roedisres e insectos como mumas, de las culates alguט parásjtos infestinalesi; y 20 -Vala: condieionce highirnicess indivictuales, l. ctı cs consecuentia del bajo nive: acconómico y deficiente grado de culturu

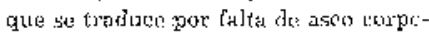

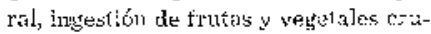
dos, cousumo do ugua Jo jotable, etc.

Considerando todas estos asprecu,; se ereyis convenicrte tealizar an estudio solsre la incidoncia de la plir:asibtosis intestioal on la ciudata de Manta, con $c !$ (1hjeto de entonital cifras numérıss che permitian una ipueciasión reij de: grach clo infestación parzstaria y slis tarateristic:as, en esta lorilidad.

\section{WATHAIALHS Y METODOS}

Para e? prosente lrabarjo se selecoionaron 300 caros, que flueron dividides

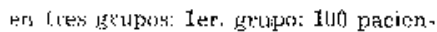
tes niñus comprentitkss on odest ascolar, al exceptión de 2 casos de lactantes rle 6 uneses de edidi estos pacientes fueron de le consultu privada y de re-

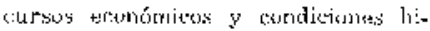
gif́nicà monos que medtamas.

2clo. grupo: 100 pucientes perteneciturtes a la clasis media (cmploados de (sfleinas), que fueron atenditos en ta con sario del Soguro Social, y de nivel coonénico y condic:anes higiérijtas mác que mentimas.

3cr. grupo: 100 cusos fjertenuciantrs is obreios tratados igralınenles on lit emsulta externa antes mencionadá.

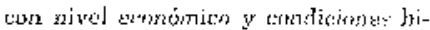
giénicas jús biem deficiontes,

En todos los pacientes se realizú un 
examen coprinaras:tario y solamente un pequeño nú:mero de ellos frut requerido para un segumblo y tercos examen; esto se debió, en grin parte, u! vortures de trithajo on el leboratoriu $y$ a la fittia de pissonul sufinicnte para realizar pxámeness seriacles. 'Todas las pacientes tomaron laxante on la noche pievia it la realización del examen astroparasitario. Solumente fos que fineron roqueridos pila un tercer exumen hat at-

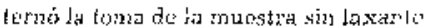

\section{'TLCNICA IDEL THXAMEN COPROPARASTTARIO}

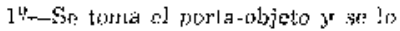
l:mpia bies, parel dopositar una gota do suero fisiplópters on tant de slas tix" tremos y ulsa gotii de solusión de Lugol, en ol otro; de kota de stuero sirve pixa exalyoribar ?as formas míviles, mientris due c] Lugnd sirve pat'a colorear los diferentes elemertos del paresisilo y das un inejor contraste.

2n-Se torma con un palillo mendadientes una pequeña parte de la muestra $y$ se la leva al sueru $y$ al targol, donde se imprimen pogueños moyj. mientos de rotaceión hasta que lá muestra tuusdo alriformetrente repartida.

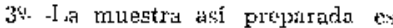
tapada con ueja laminj.le y llevada al nicroscopie, dondo se hoce lis prinerit ibsurvación eon lente de nenor aumenti para visualizar las formas más grandes; luego sc ubserva eon lomte dt mayor aument, para hates une mejor dilisentiación.

fin los casus en que esto primer axametr dio resiltado nesalivo, se tornó un al wisirs moments oflas pecjuerias pureiones de la muestra te heces paral

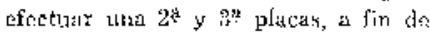
confirmals el resultadu.

Tabla I

ANAIISIS DI LOS EXAMLNES COPROPARASITATIOS OUTWNIDOS EN 'L'KES MUKSTRAS POBI.ACIONAIJS I)L' LA CIUDAD I) MLAN'A, $1964-1965$

\begin{tabular}{|c|c|c|c|c|c|c|}
\hline \multirow[t]{2}{*}{ Rutsiloltrutos } & \multicolumn{2}{|c|}{ Nis̆os } & \multicolumn{2}{|c|}{ Empleudos } & \multicolumn{2}{|c|}{ Mhrelex } \\
\hline & $\mathbf{N}^{9}$ & $\%$ & $\mathrm{~N}^{\circ}$ & 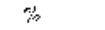 & Non & 筆: \\
\hline honoparasitados $\ldots . .$. . & 56 & 68 & \$.7 & 65.42 & 78 & 82.1 \\
\hline Poleparasidado. $\ldots \ldots \ldots$ & $26(1)$ & 32 & $28(2)$ & 35.68 (3) & 17 & 179 \\
\hline Total parasitutlos ....... & 82 & 100 & 81 & 100 & 95 & 100 \\
\hline Totâl pasitivos .... ..... & 82 & 82 & 8) & 81 & 35 & 9.5 \\
\hline l'otel neginfivos . . . . . . . & 18 & 18 & 19 & 19 & 5 & 5 \\
\hline Total cxímpons ........ & {$[00$} & 100 & 100 & 100 & $106)$ & 100 \\
\hline
\end{tabular}

(1) La usoriación más irecuente Jue te Giardia son Tricomonas,

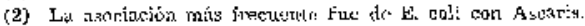

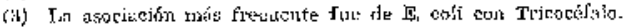




\section{Tubla II}

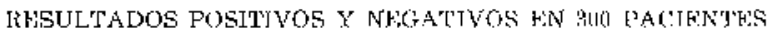

\begin{tabular}{|c|c|c|}
\hline lesuifudes & $\lambda$ & Paresentujer \\
\hline Minnugaratataless $\ldots \ldots \ldots \ldots \ldots$ & 187 & 72.48 \\
\hline 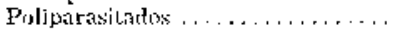 & 7.1 & $\begin{array}{c}27,51 \\
100\end{array}$ \\
\hline 'Total positiyos .............. & 2,78 & 86 \\
\hline 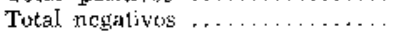 & 12 & 11 \\
\hline Total exártmenes $\ldots \ldots \ldots \ldots \ldots$ & 300 & 100 \\
\hline
\end{tabular}

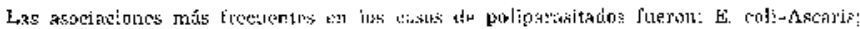
Ł. euli-Tr-inocetáalu.

\section{RESULTADOS}

Los deuter whtenidos en las 3 muesIras poblacjonales son los siguientes;

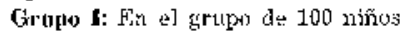
se encontro un total de 82 ctsos pusitivos; munoparasitad(ıs $\int(1,4)$ ) el f88\%: y poliparzsitados, el $32 \%$. Tubla I.

Grupo 31: lin el grupo de 100 ert- pleadus se robrevi in lutél de 81 cascsi positivis, de los suades monoparasitados fueron el 6.5.42\% $y$ poliparasitades e] $34,57 \%$ ti. 'Trabla k.

Grupo IlY: lín el grotpo de $\mathbf{1}(10$ obrerors, se oncomptró un total de 95 rasos positivos de los cuales monvpurasi. tadus fuertm el $82,10 \%$ y priliparasitados ol 17,89\%. Trebla I.

\section{T'ab)la III}

IXICUENCIA DE CADA PARASTO FN LOS 258 CASOS POSITIVOS

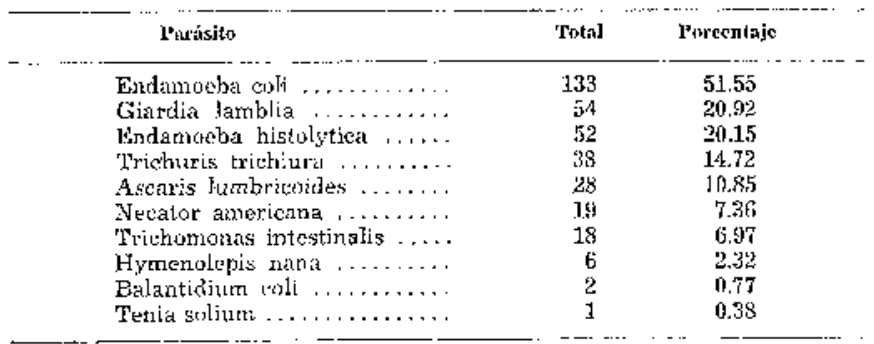


Tabla IV

\section{FRECUKNCJA DE PARASITOS DLNTRO DH CADA CRUPO (LONO Y POLIPARASITADOS)}

\begin{tabular}{|c|c|c|c|c|c|c|}
\hline \multirow[t]{2}{*}{ P'uláasiton } & \multicolumn{2}{|c|}{ Himpleatns } & \multicolumn{2}{|c|}{ Viños } & \multicolumn{2}{|c|}{ Gloreros } \\
\hline & $N$ & $\%$ & NNE & $\mathscr{F}$ & $\mathbf{N}$ & 然 \\
\hline Gijaróia lamblia $\ldots \ldots \ldots \ldots$ & 4 & 21,21 & 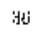 & $4: 3,90$ & 9 & 9,47 \\
\hline Endarno:lı colj $\ldots \ldots \ldots \ldots$. & 53 & $6,5,43$ & 20 & 24,39 & 60 & 63,15 \\
\hline Trichumnos ijutest:nifis ...... & - &.$\cdot$ & 14 & 37,07 & 1 & 4,21 \\
\hline 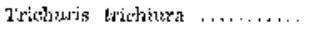 & j4 & $F 6 ;\}$ & 13 & 75,85 & 11 & $51,5 \mathrm{~T}$ \\
\hline Einclamotbir higtolytica ....... & 23 & 28,39 & $3 \mathrm{er}$ & $32,1 \%$ & $1 ?$ & 20 \\
\hline 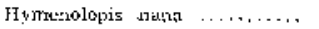 & - & --- & 5 & $t i, b y$ & l & 1,05 \\
\hline Necator amerimas .......... & 4 & $4,9 \mathrm{~s}$ & 2 & 2,43 & I3 & 13,68 \\
\hline 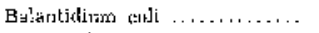 & . & $\cdots$ & 1 & 1,21 & 1 & 1,05 \\
\hline Testin soljurn $\ldots \ldots \ldots \ldots \ldots$ & $I$ & 1,23 & - & $\rightarrow$ & $\cdots$ & - \\
\hline
\end{tabular}

Do los 300 casos extudiados $25 \%$ resultaren prositjos; flo ésis motropara-

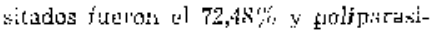
[udos e] $27,51 \%$. Luis datue restumidu\% sc elirucustran en la Tah!a Ir.

Dentro de lon: 258 casos pasitivos, se

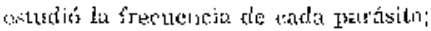
ese orden do inportencial, el primer lugat currespryste a ]a Endamoeba coli, col1 $55 \% ;$ t ] scosundon, a da Giardia lamblia, con 20,92\%; e: dereets, a la Eno damocba tistolytiru, son $20,15 \%$ la ubicaciún de los restaries partisitss se encuentra irdicada c nla 'Tubla III. El porcicnto ohiejidlo para cada parásito surressondele al totul de znoco y polipizrimsitados.

Fin lo que resparia a la frecuencia cle cada parásito dol tatal de casos positivos dentro de cadu grtupo, sc emcontró on primer lugar a lá Giardiu lasn. blis; on el grupo de empleados: 9 casos 11 ser 11.,11\%; on !ok ninus, 36 sasns con $4: 3,90 \%$; ch los obreros; 9 casoo con
9.47\%. Lus datos correxpondientes iil resto de parásitos su encuentran indicadors en la Tabla IV.

\section{ASPEC'TOS CLINICOS NAS SALIENTFS}

Debido al citlo vilal que tiouen Jos 〈liversos patrásitos del intesiju bumino, Ia sintomatologia clírica es polimorla: por lo tanto yara su arólisis se he comsiduraton lá ubiecueín mo las djversos sistonas de la.cconomín humana.

Sistema gasiso-intostinal: Casi lia to1.alidald de lus easus purasitados presenten una o varias manifestacione: dentro de este sistema. Las más importátites som:

Alowexía-Se encontró de atuerdo eon otrus auturest ${ }^{5}$, que eate sinto-

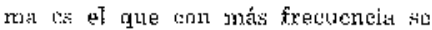
presenta, siento más manitiosto en lo: niños. 
Nátoseas y vúmitos.-Tistus as han presentadı aproximadaturale (n) una iercerit purtc de los casos y kidanente ca dos casos se prosentason vómitro de earácter alisuentieio $y$ bilioso, en forma int:yhereiblo. lin estos casos fuc necesario reciutir a li hudratat:iós parentaral; estos dos casos fueron polipttusitarkos y entambos se diayseostiró uncinariasis.

Diarrea.-L's cl síntoma rís llamaf.jvo y podríamos decir el guihin que ros indues a soiscitar ol examen copropa"dsitario; pero solamenta en eortatos casos se encontró eí verdatero cundic) disenteriforme suracterizado pox dopo-

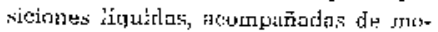
co, sangre $y$ tenesmo. Fiste cuadro ine uncontrado on crasos parasitardos de Etsdamueba histolytitu y 'I'ricocófalo.

En dos casos de lactarites pilrisitados por Necator americana sc observí une historia de tierrods cronicis (alrededise da 2 meses de duraciún) чаны evidentemente menoscabarisn el estalds stereral. Lango del tratamiento efectuado con Tetrueloretileno endió codo el cuadres patológico. restuperáludose immediatamejto dos perpueñas picicntes.

Se observó aklemás estroñimicnto ยтı pocos casos de parosijusis intestinal, s\%)bre todo los de atmebiasis, y tasi sicmJre? allermando sun episodios diareicos.

Dolor abdominal.-1)e tortos ] cos cer sos on los ruales se preseritó catc sifs." fun? se encontxá tgue la mayuría de ellos pl't'izentan un dolor dituso drel â, doinena de carícter sorta, mó́s bior. soportable; pexo también se ollsepratron casos a tos que ol dolor liue tjpo cólico y com [ifirentes ]coca]izaciuteus, siendo Tas más comumes, el mesogastrix y fosas ilík as (toner yresente que eL ol lado derecho, puedo der Jugar a conlutusion (evi un cuadro apenducular).

Hematulugia.-En lít thayoriat de los casts se observó num lígera alte. roción dể cuadro hemátieo cor şlón. los rojos illrededor de los 4 millones $y$ Ta cifrá de hemoglobita aproximuci:Laconte en los 11 gms.; en 3 casus de ututinariasis se altuvo una cilra do rojus alrededor de los 2 millnues ron 7 gisl) तe th.

Fl tipo de curemia que se procontró en la mayoria de cosos fue mierociticnhipuerómié.,

Eosinotilia._.. Se ha dicluo que la mayor parte de: las parasitosis intest:nales traen sicmpre consigro utra eosi.

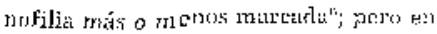
los pacientes estudiados, sóln se obilu vieron rosinotilias que nu sobrepaskrole del 5\% th pocos picicntes: on lo nuavisia do allus los eosinófilas estaljan dentro de línites normales.

Estado gencral,-Fin genteral so at1.eva puco de acuerdo a las observat:unes realizadis el todos loz casos; lo cur se encontró má́s frocuenlemente fue tula ligata astenia y una disminuc:ón

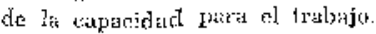

Fin los dos chses antes menejoulad pariastaris, pue Netator americana con fatcoedentes de diatreas crónites, sue derncle st: ubscrvó un gran detrimento del estado general; ainbos rsatientes prescutaron yerdaderos estados racluécelitom con peso, tallac y nivel intelettuil cinnplelainente inferjores a lo teik rejT cule inuían; se rlbservó gize la piej ern seca, los cabollos cscasos y fúáles 
$y$ la lengua con atrotia de las papjilas. Na se ubservó un suia caso que heya presentudo anasirea, $n j$ dúm siquicral edema de las extremidades in leriores.

En pocoz sátos se obscrvó una ligerela alza termicra vespertina, posib]encute debida a la asuciaciónt bacteriana secundiriza.

Sistema nerviosu, 一Los síntounas más corrumes hallados denté de estc sistema fueron la acfalea $y$ mareos, éstos tusi sicmpre ligeros y esporídicos; solo an un caso de unat phrera de 16 itros se proscntó aun rareo de ial iniet)sidad que la paciente no prdía mantenersu en posición erecla ni sentada, pur ju que tenia que atoptar el fecuibito dorsal; este mareo duró unos dos días $y$ en sill examen coproparasitario se crutertitró utucisaria.

Lals convulsiones tónico-clón kas se manifestaron ou 2 easos dr niños parasitaclos por Ascaris Jumbricides.

lín pocus vasos se entortró que presentaban escozor al rivel de las fosas nasaler $y$ en tarizimos casos: purito asal.

Sistema respiralorio,-2in este sisterna se obtuvieron pecas manifestacicines atribuidas al parasiatus intustínal; se encontró un solo caso en un indirjou poliparasitado (4 tipos de parásitos) con síntomas y sigrnos de una bronconcatnonía. Más frecuentcmentc: en niñorss se eneorztró estados br:crnquiticos y de reumonitis asociadus a paxastitosis intestinal.

\section{DISCUSION}

Iua diferenciar més notable, en cuann io se rifiere a la incidencia de un pa-

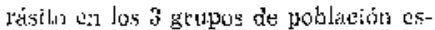
litulads, se encontró ell tocator americano. Este parásito div el 4,93\% en los emploudos, el $2,43^{\prime \prime} \%$ en los niñ mientsas en los obrerori se elova al $13,68 \%$. Esta diferencit se cxplice si se consickera que gran parte de ubrerist concurren diariancnte a su trabajo en Menta, pero tienen sul vivienda en zoras l'urales del cantón Montecristi, cono sun: El bajo y Thalle; donde reitlizan sus lareus domésticas siz calzadn.

Fste estudio se lo ba becho a base de un solo examen copruparasitorio en la mayoría de pacientes, lo que quiere decir cue el indise de murbilidad puede aunentar, en caso de que sc reatizaren exánencs seriados en los pacientes quo dícron resultado nisgativo.

Cabe señalar que en 60 s 300 rasos wo ge tancuntró un solo parasitado por Oxiuess; por lo mismn, bien valdria la perat en un estutio pusterirr; ponct nayor ateociún para tdentilicar este parásíu utilizadudo para ello cl exames del frotis perianal'.

Se auotó anteriomente dy1e las medidas saniturias tanto ambientalns como del individuo juegàn papel preporde.rente an mantonar endémica ciestas purasitosis; por lo tenlo, sería mancster tralar de corregir 0.30 finctores negativns, tratar de enseñar normas ligiéricéa al pueblo y realizar un tratumiencio antipgarasilorio tall matsa. parr. hacer disminuir la incidencia de yarasitosis intestined.

Las manitestaciones clírticas ฉıล์ îrecuentes son las referides al tracto gustro-intostina]; por la tarto, si extamus ante us paciente que presfutit anorexia, diarreas, dolor abdominil y 
si a geros mureos, debemos sospechar la presen rsia de parásitos inlestinales y zolicitor el correspondiemt! oxamen cuproparasitatis, especiulmente si sc ensuemtra sn una zolla de clima tropical.

Hiy gue setialax además gue las manifertaciones clinieas nt\} silit dependon de In clase de perésisitu que esia inlestandu al individuo, sino rambićn do] número en qué detoss se encuentrey.

1. Giardia kamblia se encortúcó que es e] parásito que alecta má: a los : i-

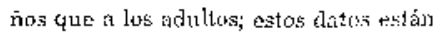
de atuexdo com los reportadus por otros

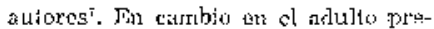
dertinia la infestación jur Fistamocba e.)]i,

I'] J3a]antidium coli es un parásito (1ute desarrolla parie de su eiclo vitut: ru cl tecto, pero que se adapta poso er e organianu himano; de allí teremos clue su incidercie en los 50 th casus. ha sido inlima.

\section{RESUMEN}

Fin 3 muestras de 1.00 individuos cado una flc la ciudad te Manta, se encontró due la incirlencia lintal de parásitos fue del 86\%. MHieniras la positividad es tast jzgual en los riño: y etuleadus, 82 y $81 \%$, respectivanentc, en los obrern: este valor Humenta al sto\%, lo que es explicable en extos individuos pur lits deficientes condicisncs sabitarias a hiǵsénicas del medin en que viven.

E1 parásitir que mayor inridencis tiene en ol tolal do las 258 k!asos pos:tivos, es la bindamoeba coli con u valor de $51,55 \%$, sigutendo en orden to. indeurtancia la Giatdia lemblis con 20.92\% : Endamoeba histolytica $20.15 \%$; Ascaris Jumbricoides $10.85 \%$.

En los abreros, lix jucidencia de los parásitos en orden Ile frexuencía zü: la sigujente: E. coli, $\mathrm{com}$ fia.15\%; E. his. tolytire 20\%; Necator $13,68 \%$.

Fin el frupo de emplexadrs: E. coli 65, $43 \%$; F. histolytien $28.39 \%$; Tricoce[a] 76,9$] \%$.

Finalmonte, en los ninos: Giardia lumblia $13,99 \%$; E. colj $24,93 \%$; tricumeงาต $13,47 \%$

\section{SLMMARY}

I1 Ilyee samples of the city w' Mantais pojoulitiom, the total incidences of parasitusis was found to be $86 \%$. While the positivity was almost. equal

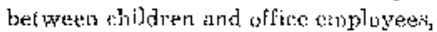
(82\% and $81 \%$ repectively), in crats. men it was loukt fhigher $(95 \%)$ due to deficient. sanitary and hygienie rotuditions of the medium in which they live.

The parasite which presented grotater incidence in the 258 positive cases considered was: Hndamveba coli, $51.55 \%$; Gierdia lamblia $20.92 \%$; Fudamocbu histolytic:i, $20.15 \%$ and $A$ scaris Lım:bricoides, $10,85 \%$.

The parasitic incidence, clussified in accordance to their frequescy of appearanse, was the following:

-In croftsmen: $F$, coli, 6.3.15\%: $P$. histolytice, 2016; Nerator, 13.68\%,

-In office employees: $E$. cols, 65.43\%: E. Jjistolytjea, 28.39\%; Trichiocephalus, $16.61 \%$.

-In childreu: G. Iasurtia, 43.90\%; E. soli, $24.43 \%$; Trichomoma, 17.07\%. 


\section{XFFFRENCIAS BIBLIOGRAFICAS}

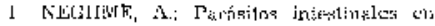

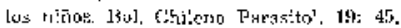
19191.

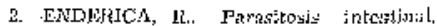
1\{:v. T.surat. Tig, y Mad. 'Tropr. 21: 61, T3lit.

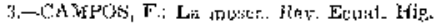
$y$ :

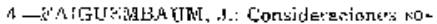

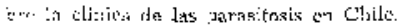

Thi. Chijeto Farasitol. 1J! 59, 1962t.

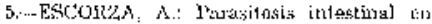
L Provincin: do Brhivar. Rev. Écual, Med.

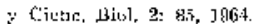

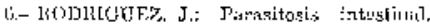

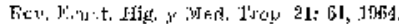

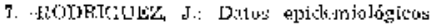

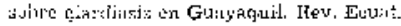

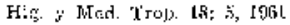

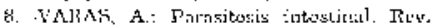

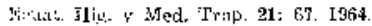

\section{PATUGINESIS JEF LA UIAERACION GASTRICA}

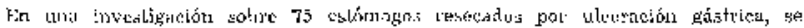

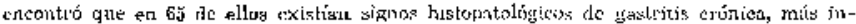

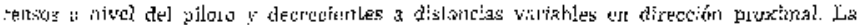

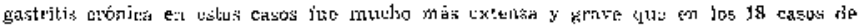
aleeració: duodenal.

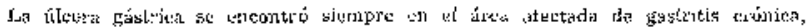

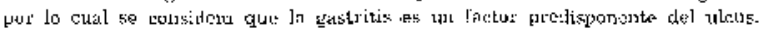

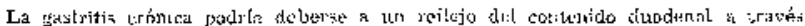
3ul pisno.

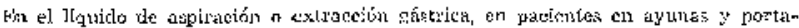

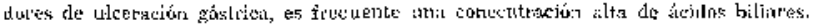

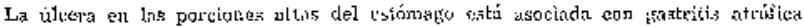

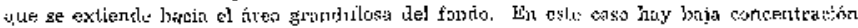

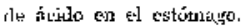

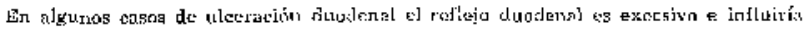

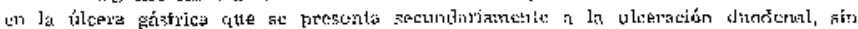
sljsulucciós.

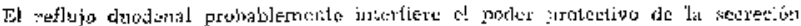

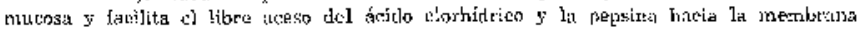

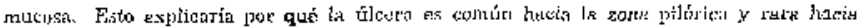
l:t zonta del caxtios. Desde luepo el reilujo duodenal no es el factor únjoo en lus casvii

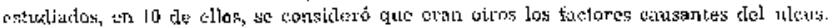

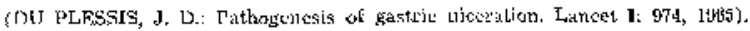

